\title{
Interobserver agreement of estimating the extent of intestinal metaplasia in patients with chronic atrophic gastritis
}

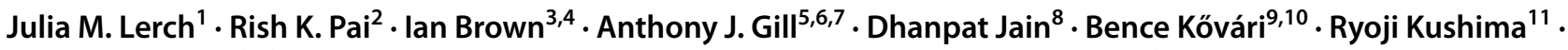 \\ Kieran Sheahan ${ }^{12,13} \cdot$ Tomas Slavik $^{14,15} \cdot$ Amitabh Srivastava $^{16} \cdot$ Gregory Y. Lauwers $^{10} \cdot$ Cord Langner $^{1}$ (i)
}

Received: 31 July 2021 / Revised: 18 November 2021 / Accepted: 23 November 2021 / Published online: 13 December 2021

(c) The Author(s) 2021

\begin{abstract}
The extent of gastric intestinal metaplasia (GIM) can be used to determine the risk of gastric cancer. Eleven international gastrointestinal expert pathologists estimated the extent of GIM on haematoxylin and eosin (H\&E)- and Alcian blue-Periodic acid Schiff (AB-PAS)-stained slides of 46 antrum biopsies in 5\% increments. Interobserver agreement was tested with the intraclass correlation coefficient (ICC). Correlation between standard deviation and extent of GIM was evaluated with the Spearman correlation. The interobserver agreement was very good (ICC $=0.983,95 \%$ confidence interval (CI) $0.975-0.990)$. The use of AB-PAS did not increase the agreement (ICC $=0.975,95 \%$ CI $0.961-0.985)$. Cases with a higher amount of metaplastic epithelium demonstrated a higher standard deviation ( $\mathrm{rs}=0.644 ; p<0.01)$, suggesting lower diagnostic accuracy in cases with extensive GIM. In conclusion, estimating the extent of GIM on H\&E-stained slides in patients with chronic atrophic gastritis can be achieved satisfactorily with high interobserver agreement, at least among international expert gastrointestinal pathologists.
\end{abstract}

Keywords Chronic atrophic gastritis · Intestinal metaplasia $\cdot$ Interobserver agreement · Gastric precancerous lesion

Cord Langner

cord.langner@medunigraz.at

1 Diagnostic and Research Institute of Pathology, Diagnostic and Research Center for Molecular BioMedicine, Medical University of Graz, Neue Stiftingtalstraße 6, 8010 Graz, Austria

2 Department of Laboratory Medicine and Pathology, Mayo Clinic Arizona, Scottsdale, AZ, USA

3 Envoi Specialist Pathologists, Brisbane, Queensland, Australia

4 Faculty of Medicine, University of Queensland, Brisbane, Queensland, Australia

5 Cancer Diagnosis and Pathology Group, Kolling Institute of Medical Research, Royal North Shore Hospital, St Leonards, New South Wales, Australia

6 New South Wales Health Pathology, Department of Anatomical Pathology, Royal North Shore Hospital, St Leonards, New South Wales, Australia

7 Sydney Medical School, University of Sydney, Sydney, New South Wales, Australia
8 Department of Pathology, Yale University School of Medicine, New Haven, CT, USA

9 Department of Pathology, University of Szeged, Szeged, Hungary

10 H. Lee Moffitt Cancer Center \& Research Institute, Tampa, FL, USA

11 Department of Pathology, Shiga University of Medical Science, Shiga, Japan

12 Department of Pathology \& Centre for Colorectal Disease, St. Vincent's University Hospital, Dublin, Ireland

13 University College Dublin, Dublin, Ireland

14 Ampath Pathology Laboratories, Pretoria, South Africa

15 Department of Anatomical Pathology, Faculty of Health Sciences, University of Pretoria, Pretoria, South Africa

16 Department of Pathology, Brigham and Women's Hospital, Boston, MA, USA 


\section{Introduction}

Gastric atrophy (GA) and gastric intestinal metaplasia (GIM) have been identified as precancerous lesions suitable for risk stratification for gastric cancer. Different staging systems which require, at minimum, biopsies of both antrum and corpus, have been proposed to define the extent of GA and GIM, such as the Operative Link for Gastritis Assessment (OLGA) [1] or the Operative Link on Gastric Intestinal Metaplasia (OLGIM) [2].

For individuals with extensive GIM, defined as GIM involving both antrum and corpus, an approximately twofold increased risk of neoplastic progression was reported compared to individuals with limited GIM [3]. While individuals with OLGIM III-IV are at high risk of early gastric neoplasia, individuals with OLGIM II were denominated as intermediate risk patients [4].

Notably, the yield of GIM may be higher when multiple biopsies are sampled, indicating that patients with a higher total number of biopsies may have a higher probability to be classified as patients with extensive GIM, that is, as "patients at risk" [5]. Therefore, scoring systems that are independent of the number of biopsies have been applied, referring either to the relative number of biopsies involved by GIM [6] or to the percentage of mucosa involved by GIM [7-9]

Our study, which includes a group of international expert gastrointestinal pathologists, aimed to evaluate the interobserver agreement in estimating the overall percentage of mucosa involved by GIM and to identify parameters with potential impact on the assessment.

\section{Material and methods}

\section{Cases}

The study included antral biopsies from 46 patients (20 females and 26 males with a mean age of 65.8 years; median 69 , range $27-87$ ) with chronic atrophic gastritis, diagnosed at the Institute of Pathology, Medical University of Graz, Austria. All biopsies had been obtained based on Sydney criteria, that is, targeting the lesser and greater curvature, excluding the normal gastroduodenal transitional mucosa. It may be of note that corpus and/or fundus biopsies, which had been submitted in separate vials, lacked GIM in all cases and were therefore not part of the evaluation.
Since Austria is a country with a low prevalence of Helicobacter pylori, resulting in a low incidence of GIM in general (with a low proportion of mucosal surface involved), we selected the study sample in order to enrich for cases with a high amount of GIM. All samples were stained with haematoxylin and eosin (H\&E) and with Alcian Blue-Periodic acid Schiff (AB-PAS) and were scanned thereafter (Pannoramic 1000 Whole-Slide Scanner, 3D Histech Ltd., Budapest, Hungary).

\section{Pathologists}

Eleven international expert gastrointestinal pathologists participated in the study. Access to scanned slides was provided by an electronically transferred web link. The assessment was performed independently (blinded to endoscopic data) on dynamic images (3D Histech Ltd. Case Viewer, Budapest, Hungary). Specifically, the pathologists were asked to estimate the overall percentage of mucosa involved by GIM in 5\% increments, that is, across all biopsies included within a given sample.

\section{Statistical analysis}

Numerical variables are presented as mean, median and range. The interobserver agreement was assessed by applying the intraclass correlation coefficient (ICC), which is used to measure the degree of agreement for continuous variables for different observers when assessing the same cases [10]. The calculation is based upon a two-way mixed model and absolute agreement. For interpretation, the scheme introduced by Altman (1991) was used: an ICC value $\leq 0.20$ suggests poor agreement, $0.21-0.40$ fair agreement, $0.41-0.60$ moderate agreement, $0.61-0.80$ good agreement, and $>0.80$ very good agreement, respectively [11].

Correlation between the standard deviation and the extent of GIM, defined by the mean score of GIM of the eleven observers, and the number of biopsy pieces per slide was evaluated by applying the Spearman correlation. Finally, a regression analysis was performed to establish a model that allows the prediction of the standard deviation from the extent of GIM. To account for non-consistent scattering in our dataset, we performed adjustment with heteroscedasticityconsistent standard error estimators [12].

All statistical operations were performed using IBM SPSS Statistics Version 26, provided by the Medical University of Graz. $P$-values were two-sided, and values $<0.05$ were considered statistically significant. 


\section{Results}

\section{Interobserver agreement}

The mucosa of the 46 cases was involved by GIM in different quantities, with mean values of $29 \%$ (range 6.8-82.7\%) for H\&E-stained and 25\% (range $6.4-81.7 \%$ ) for AB-PAS-stained slides. Mean values for individual observers ranged from 23.3 to $33 \%$ for $\mathrm{H} \& \mathrm{E}$-stained and 15.3 to $33.8 \%$ for $\mathrm{AB}$ PAS-stained slides. The interobserver agreement was very good, with an ICC value of $0.983(95 \%$ confidence interval (CI) 0.975-0.990) for H\&Estained slides and 0.975 (95\% CI $0.961--0.985$ ) for AB-PAS-stained slides, respectively. Thus, the use of AB-PAS did not increase agreement. Table 1 shows the interobserver correlation matrix of the eleven pathologists for H\&E-stained slides.

\section{Correlation between the standard deviation and potential impact factors}

The number of biopsy pieces per slide ranged from 1 to 6 (mean 2.7, median 2). No correlation between the standard deviation and the number of biopsy pieces was observed on H\&E-stained slides $(p=0.059)$. The six cases with the lowest standard deviation had a mean biopsy number of 3.0 (median 2.5 , range 2-6) whereas the six cases with the highest standard deviation had a mean biopsy number of 2.3 (median 2.5, range 1-4).
Table 1 Interobserver correlation matrix for $\mathrm{H} \& \mathrm{E}$ stained slides
Fig. 1 Histology of a case with high interobserver agreement (mean extent of gastric intestinal metaplasia of $10.5 \%$, standard deviation of $4.2 \%$ ) at low (a) and high (b) magnification. Histology of a case with low interobserver agreement (mean extent of gastric intestinal metaplasia of $67.7 \%$, standard deviation of $25.5 \%$ ) at low (c) and high (d) magnification

\begin{tabular}{llllllllllll}
\hline & $\# 1$ & $\# 2$ & $\# 3$ & $\# 4$ & $\# 5$ & $\# 6$ & $\# 7$ & $\# 8$ & $\# 9$ & $\# 10$ & $\# 11$ \\
\hline$\# 1$ & 1.000 & 0.966 & 0.920 & 0.958 & 0.882 & 0.923 & 0.965 & 0.874 & 0.830 & 0.947 & 0.742 \\
$\# 2$ & 0.966 & 1.000 & 0.927 & 0.953 & 0.871 & 0.944 & 0.949 & 0.901 & 0.817 & 0.952 & 0.717 \\
$\# 3$ & 0.920 & 0.927 & 1.000 & 0.916 & 0.846 & 0.882 & 0.915 & 0.880 & 0.790 & 0.934 & 0.679 \\
$\# 4$ & 0.958 & 0.953 & 0.916 & 1.000 & 0.869 & 0.902 & 0.934 & 0.894 & 0.778 & 0.924 & 0.749 \\
$\# 5$ & 0.882 & 0.871 & 0.846 & 0.869 & 1.000 & 0.851 & 0.860 & 0.893 & 0.669 & 0.851 & 0.692 \\
$\# 6$ & 0.923 & 0.944 & 0.882 & 0.902 & 0.851 & 1.000 & 0.930 & 0.866 & 0.802 & 0.911 & 0.691 \\
$\# 7$ & 0.965 & 0.949 & 0.915 & 0.934 & 0.860 & 0.930 & 1.000 & 0.876 & 0.804 & 0.961 & 0.723 \\
$\# 8$ & 0.874 & 0.901 & 0.880 & 0.894 & 0.893 & 0.866 & 0.876 & 1.000 & 0.725 & 0.901 & 0.677 \\
$\# 9$ & 0.830 & 0.817 & 0.790 & 0.778 & 0.669 & 0.802 & 0.804 & 0.725 & 1.000 & 0.814 & 0.722 \\
$\# 10$ & 0.947 & 0.952 & 0.934 & 0.924 & 0.851 & 0.911 & 0.961 & 0.901 & 0.814 & 1.000 & 0.674 \\
$\# 11$ & 0.742 & 0.717 & 0.679 & 0.749 & 0.692 & 0.691 & 0.723 & 0.677 & 0.722 & 0.674 & 1.000 \\
\hline
\end{tabular}

a

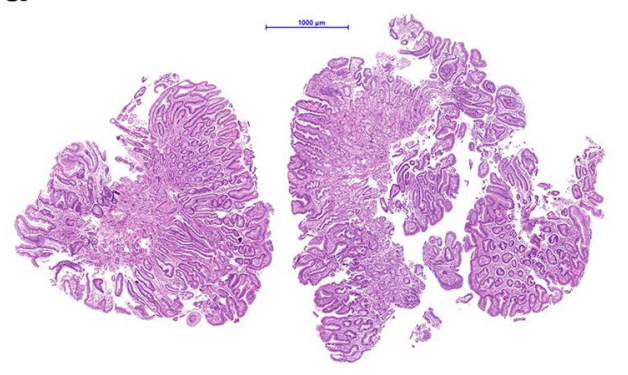

C

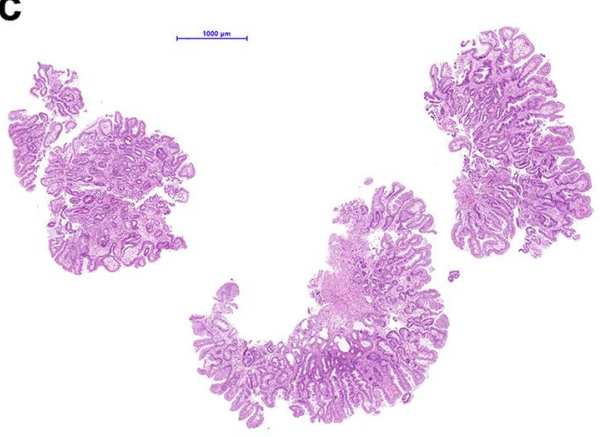

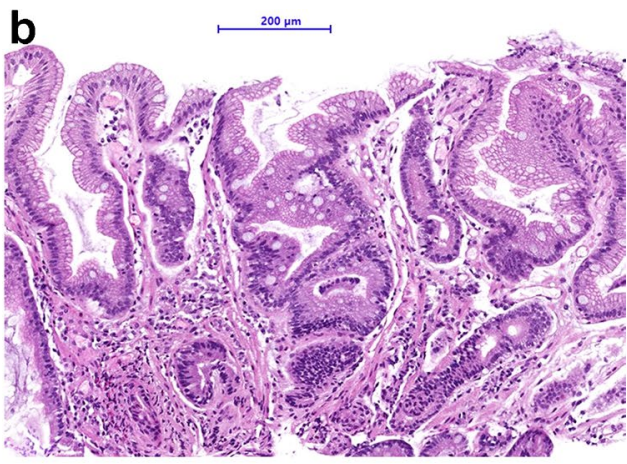

d ${ }^{200 \mathrm{~mm}}$

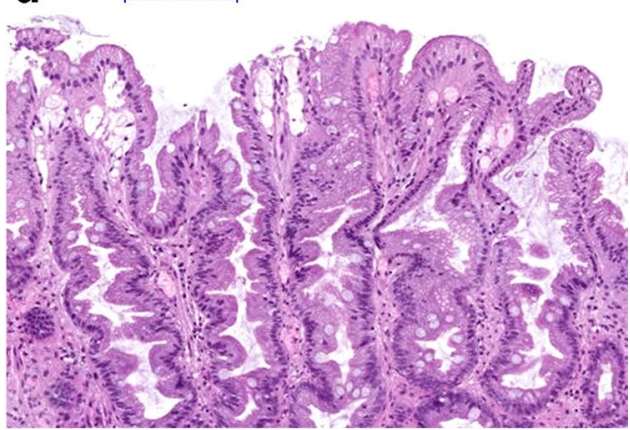




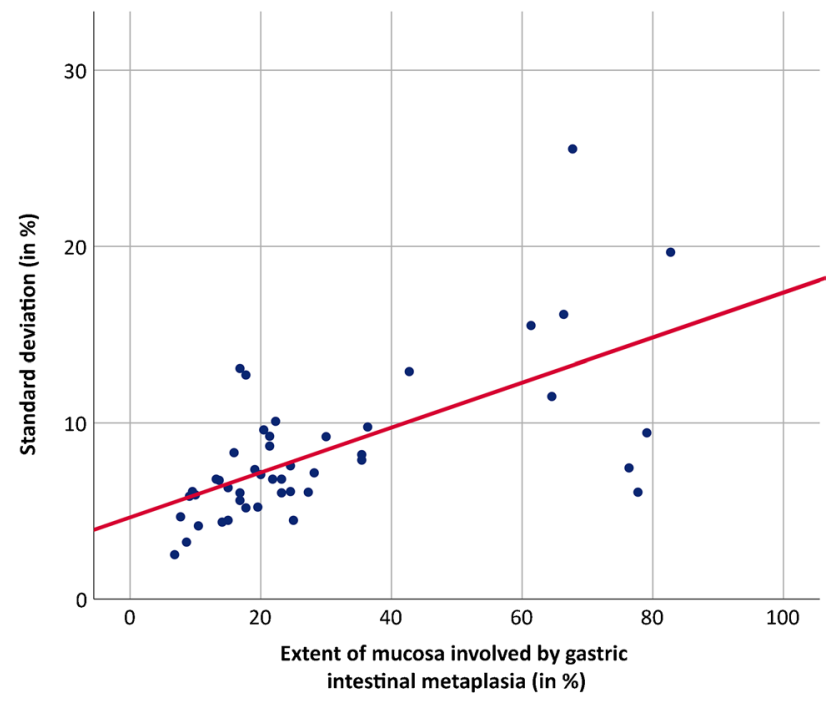

Fig. 2 Correlation between the extent of gastric intestinal metaplasia (GIM assessed on H\&E-stained slides, in \%) and the standard deviation (SD, in \%): The SD increases with the amount of metaplastic epithelium identified on the mucosal surface $(\mathrm{SD}=4.568+0.127 \times$ extent $\mathrm{GIM})$

Cases with a higher amount of metaplastic epithelium had a higher standard deviation. The significant positive association between both parameters was verified by applying the Spearman correlation, $r s=0.644(p<0.01)$. A simple linear regression was calculated to predict the standard deviation based upon the extent of GIM. According to the regression equation with $R^{2}$ of 0.403 , the standard deviation increased by $0.127(t=2.862, p<0.01)$ for each percent of the extent of $\operatorname{GIM}(\mathrm{F}(1,44)=29.749, p<0.01$; Figs. 1 and 2$)$.

\section{Discussion}

The extent of GIM in patients with chronic gastritis has prognostic relevance and identifies patients at higher risk of gastric cancer who could benefit from endoscopic surveillance [3] Current guidelines by the European Society of Gastrointestinal Endoscopy (ESGE) [13], the British Society of Gastroenterology (BSG) [14], and the American Gastroenterological Association (AGA) [15] classify the extent of GIM as "extensive," when GIM affects both antrum and corpus, requiring, at minimum, biopsies from both regions of the mucosa. For patients with extensive GIM endoscopic surveillance is recommended, while patients with GIM limited to the antrum do not need follow-up [13-15]

The number of biopsies may affect the histological diagnosis, that is, the yield of GIM may increase when multiple biopsies are obtained [5] In addition, biopsy specimens may be involved in varying quantities, with some showing only tiny foci of GIM, whereas others may show total replacement of the original mucosa. Consequently, some authors suggested alternative scoring methods, such as the (relative) number of biopsies involved by GIM [6] or the percentage of mucosa involved by GIM [7-9]

Our study proves for the first time that pathologists can estimate the extent of GIM on a semi-quantitative scale with very good agreement. The use of AB-PAS did not improve the agreement reached on H\&E-stained slides. Therefore, this stain cannot be recommended to estimate the extent of GIM for routine practice. The standard deviation did not correlate with the number of biopsy pieces per slide, indicating that semi-quantitative assessment in 5\% incremental steps can be applied irrespectively of the number of sampled biopsies.

It is of note, however, that the standard deviation increases with the amount of GIM, suggesting lower diagnostic accuracy in cases with extensive GIM. High amounts of GIM, i.e., involvement of several biopsy pieces within one sample and/or multiple foci within a single biopsy piece, may impede the estimation and may thereby have a negative impact on the quality of assessment.

Our study has strengths and limitations. Strengths include the systematic approach involving a large international group of gastrointestinal expert pathologists who analysed a large set of biopsies showing different quantities of GIM. Some may regard the lack of an independent "gold standard," e.g., provided by morphometric image analysis, as a limitation of the study. We regarded this, however, as outside the scope of our project, in particular since the routine assessment of GIM is done by usual light microscopy and not by morphometry or comparable tools. Another limitation might be the use of virtual microscopy, which bears specific technical challenges: pathologists may find it harder to move around all biopsy specimens with the same ease they do on a microscope. However, the findings in our study are still relevant in view of the expected increase in the use of virtual diagnostics in the future.

In conclusion, estimating the percental extent of GIM on H\&E-stained slides in patients with chronic atrophic gastritis can be achieved satisfactorily with high interobserver agreement, at least among international expert gastrointestinal pathologists. Our brief report provides the basis for future research in the field, e.g., by expanding the evaluation to general pathologists in a nation-wide setting, and for potential implementation of percental GIM assessment in the respective guidelines on gastric precancerous lesions.

Author contribution Cord Langner, Julia M. Lerch and Gregory Y. Lauwers designed the study. Rish K. Pai, Ian Brown, Anthony J. Gill, Dhanpat Jain, Bence Kővári, Ryoji Kushima, Kieran Sheahan, Tomas Slavik, Amitabh Srivastava, Gregory Y. Lauwers and Cord Langner performed the histological analysis. Julia M. Lerch, Rish K. Pai, Gregory Y. Lauwers and Cord Langner: analysed and interpreted the 
data. Julia M. Lerch and Cord Langner wrote the manuscript. Rish K. Pai, Ian Brown, Anthony J. Gill, Dhanpat Jain, Bence Kővári, Ryoji Kushima, Kieran Sheahan, Tomas Slavik, Amitabh Srivastava, Gregory Y. Lauwers revised the manuscript critically for important intellectual content and approved the final version of the article.

Funding information Open access funding provided by Medical University of Graz.

Data availability The datasets generated during and/or analyzed during the current study are available from the corresponding author on reasonable request.

Code availability Not applicable.

\section{Declarations}

Ethics approval Institutional Review Board approval was obtained from the Ethics Committee of the Medical University of Graz, Austria (EK 33-444 ex20/21). This study was performed in accordance with the Declaration of Helsinki.

Consent to participate Not applicable.

Consent for publication Informed consent for publication was obtained from all authors.

Conflict of interest The authors declare no competing interests.

Open Access This article is licensed under a Creative Commons Attribution 4.0 International License, which permits use, sharing, adaptation, distribution and reproduction in any medium or format, as long as you give appropriate credit to the original author(s) and the source, provide a link to the Creative Commons licence, and indicate if changes were made. The images or other third party material in this article are included in the article's Creative Commons licence, unless indicated otherwise in a credit line to the material. If material is not included in the article's Creative Commons licence and your intended use is not permitted by statutory regulation or exceeds the permitted use, you will need to obtain permission directly from the copyright holder. To view a copy of this licence, visit http://creativecommons.org/licenses/by/4.0/.

\section{References}

1. Rugge M, Meggio A, Pennelli G, Piscioli F, Giacomelli L, De Pretis G, Graham DY (2007) Gastritis staging in clinical practice: the OLGA staging system. Gut 56:631-636. https://doi.org/10. 1136/gut.2006.106666

2. Capelle LG, de Vries AC, Haringsma J, Ter Borg F, de Vries RA, Bruno MJ, van Dekken H, Meijer J, van Grieken NC, Kuipers EJ (2010) The staging of gastritis with the OLGA system by using intestinal metaplasia as an accurate alternative for atrophic gastritis. Gastrointest Endosc 71:1150-1158. https://doi.org/10.1016/j. gie.2009.12.029

3. Gawron AJ, Shah SC, Altayar O, Davitkov P, Morgan D, Turner K, Mustafa RA (2020) AGA Technical Review on Gastric Intestinal Metaplasia-Natural History and Clinical Outcomes. Gastroenterology 158:705-731. https://doi.org/10.1053/j.gastro.2019.12. 001
4. Lee JWJ, Zhu F, Srivastava S, Tsao SK, Khor C, Ho KY, Fock KM, Lim WC, Ang TL, Chow WC, So J, Koh CJ, Chua SJ, Wong A, Rao J, Lim LG, Ling KL, Chia CK, Ooi CJ, Rajnakova A, Yeoh KG (2021) Severity of gastric intestinal metaplasia predicts the risk of gastric cancer: a prospective multicentre cohort study (GCEP). Gut. https://doi.org/10.1136/gutjnl-2021-324057

5. de Vries AC, Haringsma J, de Vries RA, Ter Borg F, van Grieken NC, Meijer GA, van Dekken H, Kuipers EJ (2010) Biopsy strategies for endoscopic surveillance of pre-malignant gastric lesions. Helicobacter 15:259-264. https://doi.org/10.1111/j.1523-5378. 2010.00760.x

6. Cassaro M, Rugge M, Gutierrez O, Leandro G, Graham DY, Genta RM (2000) Topographic patterns of intestinal metaplasia and gastric cancer. Am J Gastroenterol 95:1431-1438. https://doi.org/10. 1111/j.1572-0241.2000.02074.x

7. Tava F, Luinetti O, Ghigna MR, Alvisi C, Perego M, Trespi E, Klersy C, Fratti C, Fiocca R, Solcia E (2006) Type or extension of intestinal metaplasia and immature/atypical "indefinite-fordysplasia" lesions as predictors of gastric neoplasia. Hum Pathol 37:1489-1497. https://doi.org/10.1016/j.humpath.2006.05.012

8. Zullo A, Hassan C, Romiti A, Giusto M, Guerriero C, Lorenzetti R, Campo SM, Tomao S (2012) Follow-up of intestinal metaplasia in the stomach: When, how and why. World J Gastrointest Oncol 4:30-36. https://doi.org/10.4251/wjgo.v4.i3.30

9. Pennelli G, Grillo F, Galuppini F, Ingravallo G, Pilozzi E, Rugge M, Fiocca R, Fassan M, Mastracci L (2020) Gastritis: update on etiological features and histological practical approach. Pathologica 112(3):153-165. https://doi.org/10.32074/1591-951X-163

10. Shrout PE, Fleiss JL (1979) Intraclass correlations: uses in assessing rater reliability. Psychol Bull 86:420-428. https://doi.org/10. 1037//0033-2909.86.2.420

11. Altman DG (1991) Practical statistics for medical research. Chapman and Hall, London

12. Hayes AF, Cai L (2007) Using heteroskedasticity-consistent standard error estimators in OLS regression: an introduction and software implementation. Behav Res Methods 39:709-722. https:// doi.org/10.3758/bf03192961

13. Pimentel-Nunes P, Libânio D, Marcos-Pinto R, Areia M, Leja M, Esposito G, Garrido M, Kikuste I, Megraud F, MatysiakBudnik T, Annibale B, Dumonceau JM, Barros R, Fléjou JF, Carneiro F, van Hooft JE, Kuipers EJ, Dinis-Ribeiro M (2019) Management of epithelial precancerous conditions and lesions in the stomach (MAPS II): European Society of Gastrointestinal Endoscopy (ESGE), European Helicobacter and Microbiota Study Group (EHMSG), European Society of Pathology (ESP), and Sociedade Portuguesa de Endoscopia Digestiva (SPED) guideline update 2019. Endoscopy 51:365-388. https://doi.org/ 10.1055/a-0859-1883

14. Banks M, Graham D, Jansen M, Gotoda T, Coda S, di Pietro M, Uedo N, Bhandari P, Pritchard DM, Kuipers EJ, Rodriguez-Justo M, Novelli MR, Ragunath K, Shepherd N, Dinis-Ribeiro M (2019) British Society of Gastroenterology guidelines on the diagnosis and management of patients at risk of gastric adenocarcinoma. Gut 68:1545-1575. https://doi.org/10.1136/gutjnl-2018-318126

15. Gupta S, Li D, El Serag HB, Davitkov P, Altayar O, Sultan S, Falck-Ytter Y, Mustafa RA (2020) AGA Clinical Practice Guidelines on Management of Gastric Intestinal Metaplasia. Gastroenterology 158:693-702. https://doi.org/10.1053/j.gastro.2019.12. 003

Publisher's note Springer Nature remains neutral with regard to jurisdictional claims in published maps and institutional affiliations. 